\title{
Conception Magico- Religieuse Des Agressions Sexuelles Et Du Viol Des Filles De 04 Mois A 18 Ans A L'ouest De La Côte D'ivoire (Duékoué Et Guiglo)
}

\author{
Koko Lucie N'goran \\ Ismaila Bakayoko \\ Agbadou Nakpon Joceline-Boli \\ Enseignant-chercheur, Maître-assistante, UFR Criminologie, \\ Université Félix Houphouët-Boigny Abidjan, Côte d’Ivoire
}

doi: 10.19044/esj.2016.v12n2p193 URL:http://dx.doi.org/10.19044/esj.2016.v12n2p193

\begin{abstract}
This article aims to study the sexual aggression and rape of minors in the western region of Côte d'Ivoire. The results show that as any deviancy, rape is a phenomenon which is explained by the imaginary conception of individuals and sociocultural representations design (see the designs put). Thus, acceptance and trivialization of violent sexual act or not, the submission idea of male domination and the negative attitude towards women are the key variables in explaining the rape. The choice of amicable settlements contributes to the aggravation of the phenomenon.
\end{abstract}

Keywords: Violence, Rape, fantasy, sociocultural representations, impunity

\section{Résumé}

Cet article a pour objet d'étudier les agressions sexuelles et le viol des mineures dans la région de l'ouest de la Côte d'Ivoire. Les résultats montrent que comme toute déviance, le viol est un phénomène qui s'explique par la conception imaginaire des individus, conception basée sur les représentations socioculturelles (voir les conceptions à mettre). Ainsi, l'acceptation et la banalisation de l'acte sexuel violent ou non, l'acceptation de la domination masculine et l'attitude négative envers les femmes sont les variables déterminantes dans l'explication du viol. Le choix des règlements à l'amiable concourent à l'évolution du phénomène.

Mots clés : Agressions sexuelles, Viol, conception imaginaire, représentations socioculturelles, impunité 


\section{Introduction}

Le viol selon les juridictions pénales se définit comme «tout acte de pénétration sexuelle, de quelque nature qu'il soit, commis sur la personne d'autrui par violence, contrainte, menace ou surprise », une infraction punie par le code pénal ivoirien en son article 354. Le viol est un acte que les hommes veulent passer sous silence. Il reste toujours un sujet tabou, une pratique qui demeure un objet peu étudié par les sciences humaines et sociales et pourtant, l'un des crimes les plus réprouvés par l'opinion (Ouimet, Guay et Proulx, 2000). Au cours des décennies, les théories explicatives du viol ont tenté d'éclaircir les comportements des violeurs ainsi que les facteurs qui les amènent au viol. Ainsi, de nombreuses études psychologiques et psychopathologies s'accordent sur le rôle de la personnalité antisociale comme facteur de causalité ou comme facteur facilitateur. A cet effet, trois facteurs explicatifs privilégiés sont mis en exergue par les études psychologiques sur les auteurs de violences sexuelles : facteurs développementaux; psychologiques; situationnels. Ces études montrent qu'il n'y a pas de délinquant sexuel type et que l'abuseur sexuel ne peut être défini sous une unique structure de personnalité, fut-elle pathologique, ou que l'acte sexuel soit compris comme la traduction symptomatique d'une maladie mentale spécifique (Hamon, 1999). Reste pourtant que le passage à l'acte, chez les abuseurs sexuels, peut être perçu comme la traduction d'un trouble de l'identité, produit par divers manques. Ainsi, des facteurs de préférence sexuelle ou d'absence de préférence pour les contacts entre homme et femme adulte non consentante (Barlow et Abel, 1976), des facteurs d'intolérance à la frustration (entraînent des comportements sexuels coercitifs ainsi que l'hostilité), le narcissisme (un intérêt exclusif pour les besoins immédiats et un manque de sensibilité au vécu de l'autre) et la colère constituent les motivations pour rendre compte de la personnalité du violeur. Le modèle de Hall, Graham et Shepherd (1991) met l'accent sur l'hétérogénéité des violeurs et récence quatre facteurs: une excitation sexuelle pour le viol, des distorsions cognitives qui justifient le viol, de l'hostilité face aux femmes et une personnalité antisociale (Ouimet, Guay et Proulx, 2000). Ces facteurs relèvent qu'une faible tolérance à la frustration permet le recours au viol pour résoudre les conflits interpersonnels. Somme toute, les résultats de ces études indiquent que la quasi-totalité des violeurs présentent des troubles de la personnalité et que selon la nature de ces troubles, les viols commis peuvent présenter divers degrés de violence. Proulx, Aubut, Perron et McKibben (1994) insistent sur la diversité des violeurs, lesquels présentent des profils de personnalité qui sont en relation directe avec le scénario du délit. Les liens entre les troubles de personnalité et le viol peuvent être de nature étiologique (troubles de personnalité en tant que variables causales du viol), être caractérisés par des 
liens de modulation du délit (lien entre les troubles de personnalité et le niveau de violence lors du délit) et conduire au choix de la victime et à la récidive. Ce qui conduit à une multitude de formes de viol que l'on peut classer en fonction des liens d'affectivité ou des relations entre les protagonistes (Le Goaziou, Mucchielli, 2010). Les enquêtes de victimologie montrent ainsi que le viol est avant tout un crime de proximité. Les deux tiers ou les trois quarts des viols, suivant les enquêtes, se déroulent dans des cercles d'interconnaissance affective ou relationnelle.

Il apparaît également qu'à l'instar des autres actes de violence, le viol se construirait autour de l'imaginaire et du mythe. En d'autres termes, des idées ou des préjugés tendent à dépénaliser le viol. Les résultats de plusieurs études montrent à cet effet que le mythe sur le viol (provient des attitudes et croyances généralement fausses, mais répandues et persistantes) permet de justifier l'agression sexuelle masculine. Ainsi, Chiroro, Bohner, Viki et Jarvis (2004) postulent que les individus dont l'adhésion aux mythes entourant le viol est élevée seraient davantage enclins à passer à l'acte. Ils démontrent en outre que la propension au viol, voire l'attrait induit par l'exercice de la violence sexuelle, puiserait ses racines, conscientes ou inconscientes, dans une velléité de domination abusive. Celle-ci servirait opportunément à exonérer l'auteur d'un viol de toute responsabilité, quitte à reporter le fardeau du mal engendré sur la seule victime. L'excitation sexuelle du violeur s'avérerait donc essentiellement subordonnée à la densité de son emprise sur sa victime. Cette dépénalisation de la responsabilité des auteurs dans le cas du mythe apparaît dans les études de Winkel et Kleuver (1997) qui en mettant l'accent sur la différence de points de vue sur les idées fausses sur le viol, visent à relever que les victimes de viol sont les premières responsables de leur sort. Les statistiques et les recherches révèlent une tendance pour les victimes à se confronter à des préjugés récurrents altérant leur capacité et leur droit à porter plainte ou à simplement faire connaître leur souffrance en se culpabilisant. Selon Puiseau (2012), « une explication au refus des victimes de porter plainte contre leur agresseur proviendrait d'un déni passif du viol. De nombreuses victimes croient que le viol qu'elles ont subi ne correspond pas aux caractéristiques de ce qu'elles pensent être un "vrai viol ». Elles se croient responsables car la société leur fait savoir qu'elles ont soit provoqué soit voulu la relation. Les travaux de Koss, Goodman, Browne, Fitzgerald, Keita et Russo puis ceux de Hamberger, Lohr, Bonge et Tolin (Ouimet, Guay et Proulx, 2000), regroupent en trois catégories les raisons qui poussent les victimes à être passive : Il ne s'est rien produit (l'idée que les femmes accusent souvent les hommes à tort de viol); Elle l'a voulu ou elle a aimé (l'idée qu'une femme qui dit "non » pense " oui », que la violence est sexuellement excitante pour les femmes, que la victime aurait pu résister si vraiment elle n'était pas consentante) et l’idée 
qu’elle l'a mérité (justifier par le fait qu'elle était habillée de manière trop sexy ou qu'elle marchait seule la nuit).

Ces facteurs, bien qu'importants n'indiquent cependant pas les raisons de l'évolution du viol des filles mineures de 04 mois à moins de 18 ans dans les communes de Duékoué et de Guiglo, même si la domination abusive et l'emprise de l'autorité sur les victimes s'avèrent être prouvées par les études. L’intérêt pour cette étude vient du fait que le viol, construit comme "arme de guerre" a été utilisé en Côte d'Ivoire pendant les crises politiques de 2002 et 2011 par les hommes armés pour terroriser la population, briser les familles, détruire les communautés. Les institutions des Nations Unies ont relevé à cet effet plusieurs milliers de cas de femmes violées, et la fin de ces crises devrait montrer une régression du phénomène. Pourtant, l'étude que nous avons menée entre 2013 et2015 dans les communes de Duékoué et de Guiglo pour le projet du CRDI-ville sûre, laisse entrevoir que le viol persiste, plus, se focalise de plus en plus sur les mineures de moins de 18 ans. Ainsi, même si les travaux psychologiques ont largement défriché la personnalité des criminels et que les enquêtes de victimisation ont permis d'aller plus loin dans la connaissance et la mesure du viol comme phénomène social (Mucchielli, 2008), il importe de se demander, quelle est la logique psychosocio-culturelle qui conduit des individus à violer des mineures ? Et quelles sont les circonstances qui favorisent le viol des mineures?

Ces interrogations nous conduisent à formuler l'hypothèse suivante : le viol des mineures dans les communes de Duékoué et Guiglo trouve son explication dans la conception imaginaire du viol, celui-ci se présentant comme source de pouvoir mystique et de purification. L’objectif de l'étude est de déterminer les aspects psycho-socio-culturels du viol.

Deux théories de référence soutiennent l’hypothèse, la théorie du choix rationnel de Cusson (1998) et la théorie des opportunités de Cohen et Felson (1979). En effet, ces théories stipulent que comme toute action humaine, l'acte criminel est un acte de routine au même titre que les activités légales qui procède de choix pour poursuivre un but, qui peut être une tentative d'adaptation ou une solution à un problème. Cet acte qui ne peut être réalisable que dans des circonstances favorables.

\section{Methodologie}

\section{Sites et Participants}

Cette recherche a été commanditée par le Centre de Recherche et de Développement International (CRDI) du Canada dans le projet du programme « villes sûres et inclusives » dans les communes d’Abobo, Bouaké et Duékoué.

Pour l'étude spécifique du viol, les communes de Duékoué et de Guiglo ont été choisies parce qu'elles ont été le théâtre de plusieurs cas de 
viol pendant la crise post-électorale de 2011. Ces deux communes regroupent les mêmes communautés ethniques, voire les mêmes cultures. En outre, Guiglo est la commune qui abrite les institutions juridiques et où le cabinet juridique porte assistance et protection judiciaire et fait valoir les droits des personnes vulnérables qui ont leur droit bafoués.

Les investigations ont été menées sur les deux sites de 2013 à 2015 et ont pris en compte 110 participants dont 8 victimes de viol, 2 responsables du centre social, 1 responsable du cabinet juridique et judiciaire, 2 agents de la police de Duékoué 1 présidente des femmes leaders de Duékoué et ses 4 adjointes qui apportent leur expertise et leur assistance au centre social car elles sont membres du comité de survie et de lutte contre l’impunité du viol, 1 responsable de UNPOL de Duékoué, 1 fonctionnaire des Droits de l'homme ONUCI-Guiglo, 10 membres des familles de victimes de viol, et 80 individus tout venant pour comprendre la signification que l'on accorde aux agressions sexuelles.

\section{Instruments de collecte des données}

Un questionnaire qui porte sur la manifestation, la perception et les facteurs explicatifs du viol a été administré aux individus tout venants et aux familles des victimes. Un entretien semi directs avec des items sur l'existence, les facteurs, les manifestations et les victimes du phénomène a été utilisé avec les 2 responsables du centre social de Duékoué. Et un focus group avec le groupe des femmes leaders sur les mêmes items. Notons qu'au sein de ces enquêtes, nous avons accordé une attention particulière aux recherches utilisant des enquêtes de victimisation dites auto-reportée ou relevées par les victimes prises en charge par les deux services sociaux (centre social et cabinet juridique et judiciaire).

Le recueil des matériaux statistiques du centre social et du cabinet juridique et judiciaire ont été complétées par les enquêtes de terrain. Ces interviews ont permis du point de vue de l'interprétation de prendre en compte : La manifestation des différents viols, La perception globale du viol, La place des différents facteurs selon les populations

\section{Analyse des données}

Deux méthodes d’analyse des données ont été utilisées, l'analyse quantitative et l'analyse de contenu.

Pour l'analyse quantitative, nous avons eu recours aux données statistiques descriptives des deux services sociaux et aux données du terrain recueillies auprès de la population enquêtée.

L'analyse de contenu des discours a servi pour davantage rendre compte du vécu des victimes et des significations accordées au viol. 


\section{Resultats}

Les résultats portent sur quatre points: viols constatés selon les structures sociales, type de viol selon l’âge des victimes, représentations sociales du viol, activités judiciaires.

\section{Viol constate selon les structures sociales prévalence du viol de 2012 a 2015}

Les enquêtes ont montrées selon les années le nombre de cas de viol commis dans la région de Duékoué en 2012, 79 cas ; en 201356 cas ; 2014 39 cas et en 2015 (de janvier à Aout) 20 cas. Il ressort de ces résultats que le nombre de cas de viol a fortement diminué d'une année sur une autre. On peut comprendre à cet effet que les multiples campagnes de sensibilisation depuis la crise postélectorale de 2011 ont eu un impact certain dans la décroissance du phénomène. Mais cela ne reflète cependant pas la réalité car le règlement à l'amiable au sein des familles pour une cohésion sociale, le manque de confiance de la population envers les autorités policières et judiciaires, les menaces d'exclusion communautaire, la dépénalisation de la responsabilité des violeurs, la lenteur de la procédure qui engage trop de dépenses financières sont autant d'éléments qui favorisent l'augmentation du chiffre noir au détriment des chiffres officiels. Les différentes agressions signalées au cabinet juridique et judiciaire les premiers six mois de l'année 2014 sont les suivantes : Le premier semestre de l'an 2014 n'a enregistré que 12 cas de viol et 2 cas d'agressions sexuelles. De ces cas de viol, on remarque que les filles sont les plus exposées au viol (9 cas soit $75 \%$ ). Le graphique suivant met en évidence l’évolution des agressions du premier semestre des années 2013 et 2014.

Figure $n^{\circ} 1$ : graphique comparatif des cas de viol 2013 et 2014

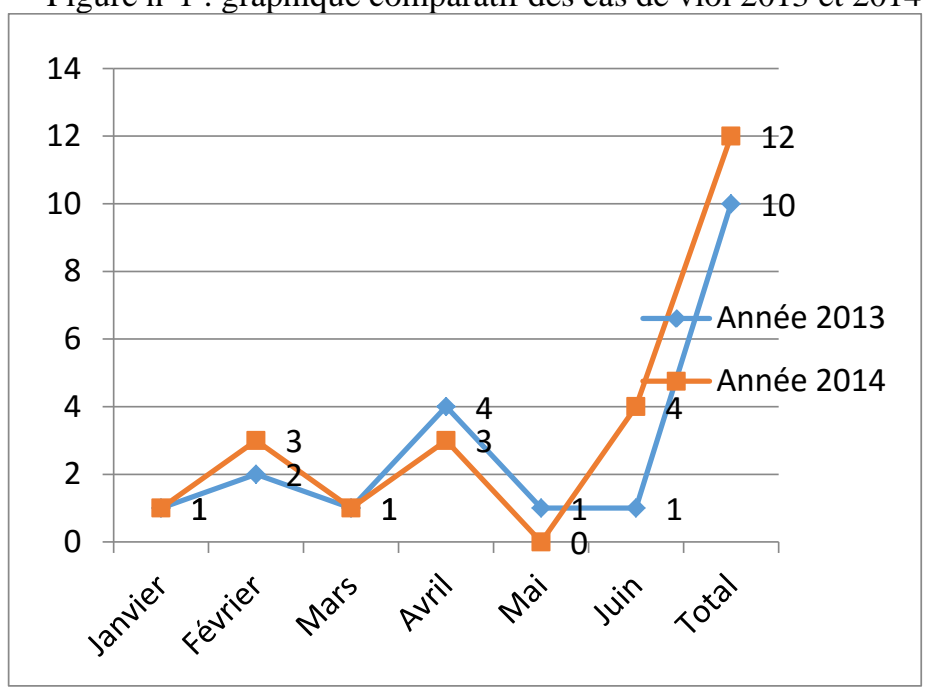

Source: Cabinet juridique et judiciaire de Guiglo 
Le graphique montre qu'il a été enregistré pour le premier semestre 2014, 12 cas de viol comparativement au premier semestre 2013 avec 10 cas de viol. De ce fait, on note une hausse des cas de viols perpétrés. Par ailleurs, en comparant les différentes périodes, il ressort que les mois de février, avril et juin enregistrent plus de cas de viols. Ainsi pendant l'année 2013, il a été enregistré en février 2 cas ; Avril 4 cas et Juin 1 cas. Par contre pendant l'année 2014 ; nous avons en Février 3 cas, Avril 3 cas et 4 cas de viol en Juin.

Ici, nous dit un des responsable du cabinet juridique, "les viols sont connus par le public mais sans pour autant attirer l'attention de la population car pour porter plainte, il faut une consultation à 2000 Francs CFA et un certificat médical qui nécessite une somme de 50000 Francs CFA. C'est ce qui décourage les victimes et encourage la récidive. Même lorsque le cabinet se saisit du cas du viol et qu'il prend en charge la victime, la famille fait pression pour arrêter la poursuite judiciaire ».

\section{Types d'agressions selon l'âge des victimes}

Les données issues des enquêtes montrent que le nombre de victimes se retrouvent dans les tranches d'âge de 6-9 ans, 10-14 ans et 15-18. Le taux le plus fort se retrouve chez les enfants âgés de 10 à 14 ans avec 5 cas soit $41,67 \%$ pour les victimes venues se présenter au cabinet pendant les six premiers mois de 2014.

Image d'une fillette de 7 mois morte suite à un viol commis par un homme de 35 ans à Duékoué le 17/03/2013 à 10 h27mn

Image d'une jeune fille violée et assassinée

à Duékoué le 18/12/2013 à $15 \mathrm{~h} 11 \mathrm{mn}$
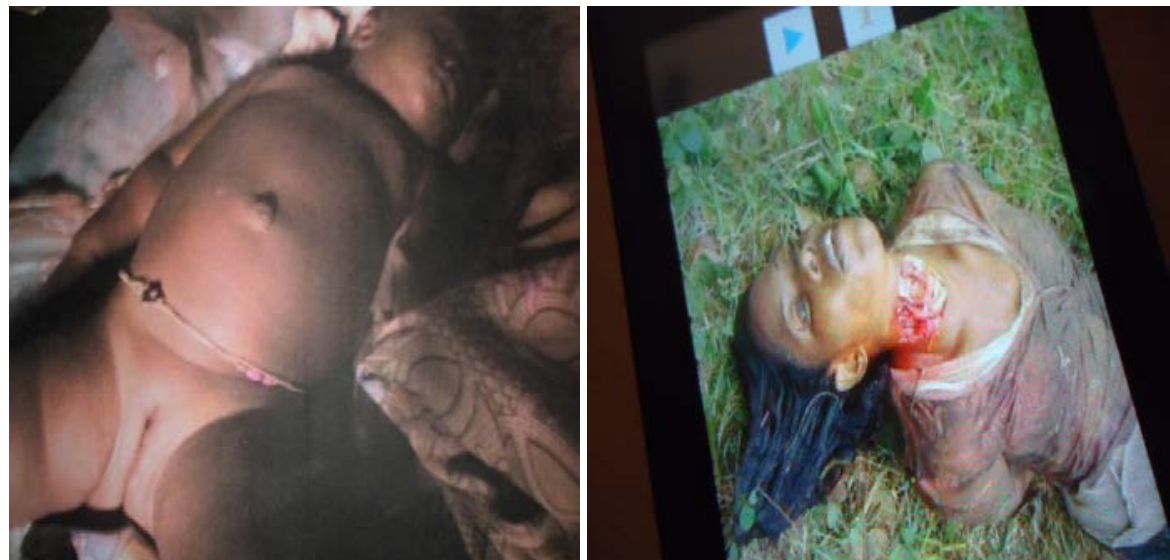

Source : UNPOL Duékoué

Tableau 4: taux de victimisation selon les années

En outre, les données du centre social relevées selon l'âge montrent que ce sont les populations âgées de 04 mois à 18 ans qui sont les victimes. 
Ainsi, nous remarquons à cet effet que 2012 (52 \%) et 2013 (80 \%) présentent les forts taux de victimisation des mineures comme le signifie les images. Les années 2014 (34 \%) et 2015 '16 \%) présentent des taux sensiblement en baisse. Est-ce dû à la réouverture de la cours d'assise de Guiglo et à la requalification de l'attentat à la pudeur (3 mois d'emprisonnent avec la fermeture de la cour d'assise) au crime (emprisonnement à vie si la victime est mineure avec l'ouverture des instances judiciaires), telle est la question que l'on se pose puisque le phénomène continue d'exister car les circonstances qui induisent le viol continue toujours d'exister.

\section{Representations sociales du viol}

La persistance du viol après la guerre tient dans l'inégalité entre les sexes ainsi que dans des constructions sociales de masculinités et féminités qui tentent à normaliser et reproduire des relations de violence entre sexe (Stanton-Jean, 2012, p 5). Les structures culturelle et sociale qui conduisent les relations au sein des communautés conduisent la reproduction des violences faites aux femmes en général et le viol en particulier. Ici nous dit la présidente des femmes leaders "La femme n'est pas considérée dans nos régions et cela se traduit par la manière dont elle est utilisée ». Même la définition donnée à l'acte de viol: "tomber sur elle" contribue à minimiser celui-ci en façonnant ainsi la tolérance sociale à l'inégalité sexuée. Ainsi, lorsqu'une fille est violée on lui demande ce qu'elle a fait pour que cela arrive. Nombre de préjugés courants sur fond de mythes tendent à parsemer le parcours des victimes d'agressions sexuelles. La suspicion, le doute, la remise en question des témoignages et l'incrédulité réactionnelle qui en résulte, renforcent la douleur ressentie par la victime, accusée souvent à tort du fait de l'agression. On lui rétorque que c'est parce qu'elle est aimée ou bien parce qu'elle-même aime cela qu'elle est violée. Le viol en quelque sorte n'a plus d'importance puisqu'il constitue un acte normal (banal) par lequel une fille doit passer et il est anormal de mettre l'agresseur en prison pour un tel acte. L'homme est en droit d'avoir une emprise totale sur le corps de la femme. Cela met à nu la vulnérabilité de la femme, ici "instrumentalisée", rendue à l'état d'objet de plaisir. Un acte que les femmes ne peuvent non pas oublier car elles vivent avec leurs bourreaux qu'elles sont obligées de côtoyer. Ainsi, comme le dit Nahoum-Grappe (2012) le viol est un crime continu dont le taux de destructivité et de production de souffrance se nourrit de la durée contrairement au crime d'assassinat où quand c'est fini c'est pour toujours. Quels peuvent être alors les facteurs qui conduisent à ces viols? 


\section{Place des différents facteurs}

La construction stéréotypée du sexe dans les productions culturelles "Guéré" (groupe ethnique de l’ouest de la Côte d’Ivoire) banalisant les rapports sexuels homme-femme, diffuse massivement des représentations perpétrant les attributions, le statut et le rôle de la femme qui n'est pas considérée comme l'égale de l’homme. Ces représentations renforcent les hommes dans leur agir et les conduisent à passer à l'acte. Plusieurs facteurs sont émis dans l'explication du viol des mineures entre autres, la reconquête d'un pouvoir mystique disparu ou altéré, la guérison d'une maladie sexuellement transmise (SIDA, MST...), la précocité sexuelle des filles.

\section{Reconquête d'un pouvoir mystique}

Comme dans toute communauté ivoirienne, la communauté "Guéré" s'est construite sur un fondement culturel qui lie le monde réel au monde surnaturel par l'instauration d'un pouvoir dévolu à une personne choisie selon les règles issues des us de la coutume. La guerre post-électorale, avec sa cohorte de violence a détruit les autorités communautaires et avec elles des postulats d'ancrage d'une puissance imaginaire qui les soutenait à toutes les épreuves. Aujourd'hui bafoués et soumis à toutes sorte d'humiliation, ces hommes autrefois admirés, car porteurs de masque ou danseurs de masque pensent reconquérir le prestige par des actes sacrificateurs, d'où des rapports sexuels forcés avec des filles encore pures (vierges). "La demande de sang recueilli au cours du viol pour faire le travail est appelé zamour » nous dit monsieur $\mathrm{X}$, un des chefs des comités de quartier. Cet individu s'installe dans une récidive et est devenu un «abuseur-violeur» tant qu'aucune solution n'est trouvée à son problème, satisfaisant ainsi ses pulsions sexuelles. Il n’est pas inquiété car soutenu par sa communauté qui devient protectrice de ses agissements en ignorant les conséquences sur le devenir des fillettes créant ainsi un espace d'impunité. Il en est de même avec le mythe de la guérison des maladies transmissibles.

\section{Guérison d'une maladie transmissible}

Perçu dans l'imaginaire de certains individus comme moyen de guérison des maladies sexuellement transmissibles, le viol perpétue l'agressivité masculine. Tout comme dans la reconquête du pouvoir, les mêmes pratiques de la pureté virginale sont indexées. En effet, dans bien des cas, les agresseurs sur recommandation de leurs guérisseurs ou marabouts pensent pouvoir guérir du SIDA ou des MST en ayant des rapports avec des mineures. Ainsi, par le truchement de l'acte sexuel, s’instaure la purification et la guérison. Le cas plus palpant est celui du viol du bébé de 7 mois soustrait par le voisin la nuit au côté de la mère endormie. 


\section{Précocité sexuelle}

Dans une société où les valeurs sociales sont de plus en plus dissolues et où les activités ludiques deviennent des moments de défoulement et de détente, les occasions de sortie deviennent des moments de chasse pour les hommes. Ainsi, l'acceptation d'une invitation à "prendre un pot" doit se terminer selon l'homme à une "coucherie". Une jeune fille qui a subi une tentative de viol nous raconte : "j'ai été invité par un jeune du quartier qui m'a vu un soir dans la rue et j'ai accepté d'aller faire le chaud avec lui. Après m'avoir raccompagné, il a voulu sortir avec moi et j'ai refusé en lui disant que c'est la première fois qu'il m'invite. Je l'ai donc laissé dans la cour et suis rentrée me coucher pensant qu'il était rentré chez lui. Mais il a attendu quelque temps pour que je m'endorme puis est rentré dans la maison car la porte n'est pas verrouillée. C'est quand il s'est couché sur moi que j'ai crié. Pris de peur il s'est échappé et à fuit le quartier jusqu'aujourd'hui. Ses parents ont demandé pardon et c'est resté comme ça ». Il ressort de ce récit que le non versus du oui de la femme qui ne peut exprimer son consentement que par la négation ne peut être accepté par les hommes. L'enquête révèle à cet effet, qu'en outre le fait de ne pas accepter le refus des jeunes filles, leur comportement provocateur, leur disponibilité et leur ingéniosité à "vider" la poche des hommes les amènent à être des victimes des hommes. Car nous disent certains enquêtés : "la précocité sexuelle des filles les amène à se mettre dans certaines situations ambigües de provocation. Ainsi, certains jeunes hommes oisifs et drogués qui n'arrivent pas par les moyens normaux à satisfaire leurs besoins sexuels profitent des attitudes de ces filles qui déjà à 10 ans et 11ans se retrouvent dans les maquis à des heures tardives

\section{Relation entre l'agresseur et la victime}

En dépit de quelques exceptions, les auteurs de viols sont quasiment toujours des hommes. Et des hommes en moyenne assez jeunes (environ 23 ans à 35 ans), qui sont en majorité dans les mêmes espaces de vie que les victimes ou qui ont même des liens de parenté à l'exception des viols commis lors des braquages. Ce faisant, il apparaît comme le souligne Le Goaziou, Mucchielli (2010), que le viol - à l'instar de l'homicide - est avant tout un crime de proximité. Les deux tiers ou les trois quarts des viols, suivant les enquêtes, se déroulent dans des cercles d'interconnaissance affective ou relationnelle. Cela peut être des copains ou des amis des victimes, des voisins ou bien encore des relations ou des connaissances, du voisinage. Et ce sont ces raisons qui conduisent souvent à suspendre les poursuites. 


\section{Activites judiciaires}

Les activités judiciaires se comprennent ici par le dépôt de plainte et la poursuite des auteurs. Cependant, avant de parler des activités judiciaires, il convient de signifier que le viol des mineures se définit comme tous «Les actes de pénétration sexuelle commis quel que soit le moyen utilisé pour y parvenir (violence, contrainte, menace ou surprise) et même lorsqu'il n'y a pas de résistance de la part de la victime ». Cela s'explique par le fait que le consentement de la mineure n'enlève pas le caractère déviant de l'acte. En effet, la mineure est présumée présomption irréfragable, et même lorsqu'elle a consenti, ce consentement n'est pas éclairé. C'est cette particularité qui distingue le viol commis sur la personne d'un enfant de celui commis sur la personne d'un adulte, l'enfant étant celui qui n'a pas atteint l'âge de dix-huit ans. Ainsi, la possibilité de porter plainte incombe aux parents qui pour une raison de cohésion sociale choisissent le règlement à l'amiable à cause des liens relationnels entre auteur et victime. Les enquêtes ont montrées combien de fois il est difficile de poursuivre les auteurs.

En effet, les données recueillies montrent qu'il existe une bonne collaboration des victimes en matière de prise en charge juridique et judiciaire. Sur douze (12) cas de viol, onze (11) des victimes ont porté plainte à la police ou à la gendarmerie, ce qui a conduit à la détention préventive de 06 auteurs. Cependant aucun des auteurs poursuivis n’a été condamné. Et c’est cela qui se constate sur le terrain car, dans la laspepiplupart des cas, les viols et autres violences sexuelles sont passées sous silence et ne sont pas portés devant les tribunaux ou les autorités compétentes, ou sont gérés de manière informelle, à ou l'amiable avec les autorités communautaires. Elles le sont ainsi, soit par crainte des:

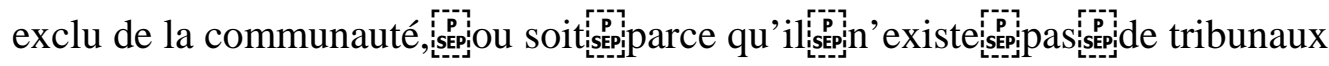
dans les zones où ont été commis lesdits actes où à cause des dépenses financières occasionnées par la poursuite judiciaire. population affirme que les des victimes et même lorsqu'elles donnent suite à ces plaintes, les agresseurs présumés ne sont pas incarcérés, ou sont libérés après une courte période de détention alors que ceux-ci vivent avec les victimes dans le même quartier. C'est ce qui occasionne les règlements à l'amiable (une somme de 15 000, de la boisson qu'une personne influente apporte pour demander pardon). En effet, nous dit les parents d'une fillette de 11 ans atteint du SIDA suite à un viol «Il est difficile pour nous de saisir les autorités ou de porter plainte car lorsque tu le fait l'agresseur arrive avant toi au quartier, il est libéré on ne 
sait pour quelles raisons. Il te nargue et de menace. Voilà pourquoi il est mieux de régler l'affaire en famille et à l'amiable. Aujourd'hui j'envoie mon même ma fille à l'hôpital pour suivre son traitement».

Ainsi, pour la plupart des poursuites des auteurs, la plate-forme (constituée du centre social, la gendarmerie, du commissariat, des médecins et de la justice) tout en portant assistance aux victimes, s'auto saisie pour enclencher les actions procédurales lorsque s’avère le constat du viol ou lorsque le viol est signalé aux autorités car la question des poursuites pénales n’est possible qu'à la suite du dévoilement ou du signalement de l'acte alors que la plupart des plaintes sont retirées par les parents.

\section{Discussion et conclusion}

Les résultats de cette étude même s’ils ne peuvent être généralisés sur les autres communes de la Côte d'Ivoire, confirment notre hypothèse, à savoir que la fréquence élevée du viol des mineures dans les communes de Duékoué et Guiglo trouve son explication dans la conception imaginaire du viol, celui-ci se présentant comme source de pouvoir mystique et de purification. Ces résultats confirment également les analyses de Chiroro, Bohner, Viki et Jarvis (2004) qui postulent que les individus qui ont une adhésion élevée aux mythes entourant le viol seraient davantage enclins au passage à l'acte.

Ces résultats confirment également les conceptions théoriques selon lesquelles, le viol comme toute autre activité humaine est choisi délibérément lorsque des opportunités sont offertes pour résoudre un problème, celui de la reconquête du pouvoir et de la guérison et ne répond donc pas toujours à des besoins sexuels (Cusson ,1998 ; Cohen et Felson, 1979).

L'étude montre en outre que la représentation du viol, phénomène de proximité se développe à cause de l'impunité des agresseurs et du choix du règlement à l'amiable car phénomène socialement accepté et aujourd'hui encore, porter plainte en cas de viol demeure une démarche rare. Le manque de moyens financiers pour porter plainte et engager les poursuites étant rares, les taux de renvois vers les instances pénales restent presqu'inexistants (Mucchielli, 2008).

Cependant, outre les facteurs individuels relevés, il est important de retenir que le contexte socio- culturel met en évidence trois variables d'importance collective: Acceptation et la banalisation de l'acte sexuelle violent ou non, Acceptation de la domination masculine et Attitude négative envers les femmes. La théorie multifactorielle intégrative qui explique l'agression sexuelle comme reposant sur l'interaction des facteurs sociaux, développementaux et situationnels (Marshall et Barbaree, 1990) se confirme. 
Il est donc opportun que la lutte contre le viol en général et celui des mineures en particulier s'articule autour des fondements socioculturels du peuple en présence dans la recherche des solutions idoines.

\section{References:}

Barlow, D.H. et Abel,G.G. (1976). Sexual deviation, in Behavior modification:principles, issues and applications, Boston, Houghton \& Mifflin,p. 341-360.

Chiroro, P., Bohner, G. Tendayi V. G. et Jarvis C. I. (2001). « Beyond rape myths: A more complex view of perceptions of rape victims », Sex roles. (en) Amy M. Buddie et Arthur G. Miller, vol. 45, $\mathrm{n}^{0} 3-4$, p. 139- 160 (DOI 10.1023/A:1013575209803) publié en avril 2004 dans le Journal of Interpersonal Violence.

Cohen, E., Felson, M. (1979). Social change and crime rate trends. A routine activity approche, American sociological review, PP. 588-608.Cusson, M. (1998). Criminologie actuelle, Paris, PUF.

Dubec, M. et De Rudder, C. (2007). Le plaisir de tuer, Paris, éditions du Seuil, p. 211, 212, 213.

Hamon, F. (1999). Délinquance sexuelle et crimes sexuels, Paris, Masson.

Kobou Bi G. S. (2008). Crise et violences basées sur le genre en côte d'ivoire résultats des études et principaux défis, Edicoms-Sarl pour le compte de l'UNFPA.

Koss, M., Goodman, L.A., Browne, A., Fitzgerald, L.F., Keita, G.P. et Russo,

N.F. (1994). No Safe Haven: Male Violence Against Women at Home, at Work, and in the Community, American Psychological Association (APA), NCJRS Abstracts Database, 344 p. (ISBN 1557982449 et 978-1557982445, OCLC 4769378274).

Le Goaziou V., Mucchielli L. (2010). Les viols jugés en cours d’assises : typologie et variations géographiques, Questions pénales, 4, 1-4.

Marshall,W.L.,\& barbareeH.E. (1990). An integrated theory of the etiology of sexual offending in Marshall, W.L., D.R.Laws et H.E. Barbaree (Eds) Handbook of sexual assault: Issues, theories, and treatment of offenders, New York Plenum Press.

Mucchielli, L. (2005). Recherche sur les viols collectifs: données judiciaires et analyse sociologique: "Les classiques des sciences sociales http://www.uqac.ca/Classiques_des_sciences_sociales/

Mucchielli, L. (2008) Une société plus violente ? Analyse socio-historique des violences interpersonnelles en France, des années 1970 à nos jours », Déviance et société, 2, 115-147.

Ouimet,M., Guay, J.-P. \& Proulx, J. (2000). Analyse de la gravité des Agressions sexuelles de femmes adultes et de ses déterminants. Revue 
internationale de criminologie et de police technique et scientifique, 53(2), 157-172.

Proulx, J., Aubut, J., Perron L., et McKibben A. (1994). Troubles de la personnalité et viol : implications théoriques et cliniques Criminologie, vol. 27, nº 2, 1994, p. 33-53. http://id.erudit.org/iderudit/017354ar.

Proulx J., Cusson M., Beauregard E., Nicole A., (dir.), 2005, Les meurtriers sexuels: Analyse comparative et nouvelles perspectives, Montréal, Presses de l’Université de Montréal.

Puiseau, B.W. (2012). What are rape myths? A European Association of Psychology and Law, Student Society Publication, P. 1-4.

Winkel F. W. et Kleuver E. D. (1997). Communication Aimed at Changing Cognitions About Sexual Intimidation: Comparing the Impact of a Perpetrator-Focused Versus a Victim-Focused Persuasive Strategy, vol. 12, Journal of Interpersonal Violence, (OCLC 4769438836), p. 513-529. 